\title{
FREE TOPOLOGICAL GROUPS AND THE PROJECTIVE DIMENSION OF A LOCALLY COMPACT ABELIAN GROUP
}

\author{
JOHN MACK, SIDNEY A. MORRIS AND EDWARD T. ORDMAN
}

ABSTRACT. It is shown that a free topological group on a $k_{\omega^{-}}$ space is a $k_{\omega}$-space. Using this it is proved that if $X$ is a $k_{\omega}$-group then it is a quotient of a free topological group by a free topological group. A corollary to this is that the projective dimension of any $k_{\omega}$-group, relative to the class of all continuous epimorphisms admitting sections, is either zero or one. In particular the projective dimension of a connected locally compact abelian group or a compact abelian group is exactly one.

1. Introduction. In [2] Graev showed that a free topological group on a compact Hausdorff space is a $k_{\omega}$-space. It was observed [6] that Graev's proof worked not only for free topological groups but also for groups with the maximum topology relative to a given compact set, and several applications to free products of topological groups were obtained [8], [9], [11], [12].

It is shown here that any group having the maximum topology with respect to a $k_{\omega}$-space generating it algebraically is itself a $k_{\omega}$-space. In particular a free topological group on a $k_{\omega}$-space is a $k_{\omega}$-space. Thus our result generalizes Graev's result and our proof, which relies on known facts about $k_{\omega}$-spaces, is easier than Graev's—or at least less delicate.

Recently Nummela [11] showed that the projective dimension of a compact abelian group, relative to the class of epimorphisms admitting sections, is exactly one. This was done by proving that a compact abelian group is a quotient group of a free abelian topological group by a free abelian topological group. The proof used Theorem 10 of [2] which provides a condition under which a subgroup of a free topological group on a compact set is a free topological group. (In general, such a subgroup is a free group but may have the wrong topology to be a free topological group.) We generalize Theorem 10 of [2] and thus conclude that the projective dimension of an abelian $k_{\omega}$-group is zero or one. In particular, the projective dimension of a connected locally compact abelian group or a compact abelian group is exactly one.

Received by the editors November 21, 1972.

AMS (MOS) subject classifications (1970). Primary 22A05, 54D50, 18G05; Secondary 18G20, 18G25, 22D05, 22B05, 22E05.

Key words and phrases. Free topological groups, $k_{\omega}$-space, projective abelian group. 
2. Notation and preliminaries. We assume familiarity with the notions of free topological groups due to Markov [4] and Graev [2]. Given a completely regular space $X$ we denote by $F G X(Z G X)$ the Graev free (free abelian) topological group on $X$.

Recall that a $k_{\omega}$-space is a Hausdorff topological space with compact subsets $X_{n}$ such that (i) $X=\bigcup_{n=1}^{\infty} X_{n}$; (ii) $X_{n+1} \supseteq X_{n}$ for all $n$; (iii) a subset $A$ of $X$ is closed if and only if $A \cap X_{n}$ is compact for all $n$. (For information on $k_{\omega}$-spaces see [5] and [13].) Whenever we say that a $k_{\omega}$-space has decomposition $X=\bigcup X_{n}$, we mean that the $X_{n}$ have properties (i), (ii) and (iii) above.

We will use the following properties of $k_{\omega}$-spaces: the direct product of two $k_{\omega}$-spaces is a $k_{\omega}$-space; a quotient space of a $k_{\omega}$-space is a $k_{\omega}$-space; a closed subspace of a $k_{\omega}$-space is a $k_{\omega}$-space; if a function $f$ defined on a $k_{\omega}$-space $X=\bigcup X_{n}$ is continuous on each $X_{n}$, then it is continuous on $X$; if $X=\bigcup X_{n}$ is a $k_{\omega}$-space then any compact subset $Y$ of $X$ is contained in some $X_{n}$; if $X=\bigcup X_{n}$ is a $k_{\omega}$-space and $Y_{1}, Y_{2}, \cdots$ is an increasing sequence of compact subsets of $X$ such that each $X_{n}$ is contained in some $Y_{m}$ then $X$ also has decomposition $X=\bigcup Y_{n}$. By way of examples, we note that every connected locally compact group and every compact space is a $k_{\omega^{-}}$ space.

In [11] Nummela introduced a concept of projective dimension for abelian topological groups. For our purposes it suffices to note that an abelian topological group is of projective dimension one if and only if it is not projective and it is a quotient group of a free abelian topological group by a free abelian topological group.

If $G$ is a group and $X$ is a subset of $G$, we denote by $G_{n}(X)$ the set of words in $G$ of length not exceeding $n$ with respect to $X$. We note that if $G$ is a Markov or Graev free topological group on the Hausdorff space $X$ then $G_{n}(X)$ is closed in $G$ for all $n$. (This fact is stated in [1] and can be proved in a similar manner to Theorem 2.2 of [6].)

We will denote the identity of any group by $e$.

If $X$ is a topological group, $F G X$ is the Graev free topological group on $X$ and $x$ and $y$ are in $X$, then we denote by $x \cdot y$ the product in $F G X$ of $x$ and $y$. We denote by $x y$ the product of $x$ and $y$ in $X$.

\section{Results.}

THEOREM 1. Let $X$ be a $k_{\omega}$-space and $G$ a Hausdorff group which is generated algebraically by $X$ and is such that the topology of $G$ is the finest group topology which induces the same topology on $X$. Then $G$ is a $k_{\omega}$-space and a subset $A$ of $G$ is closed if and only if $A \cap G_{n}(X)$ is closed in $G_{n}(X)$, for all $n$. 
Proof. Let $X=\bigcup X_{n}$ be a decomposition of the $k_{\omega}$-space $X$ into compact sets, so that $G=\bigcup X^{n}$, where the sets $X^{n}=G_{n}\left(X_{n}\right)$ are compact. We must show this is a decomposition of $G$ as a $k_{\omega}$-space.

Let $\tau$ be the given topology on $G$ and define a topology $\tau^{\prime}$ on $G$ as follows: $A$ is a closed set in $\tau^{\prime}$ if and only if $A \cap X^{n}$ is compact, for all $n$. Clearly $\tau^{\prime}$ is a Hausdorff topology and $\tau^{\prime} \supseteq \tau$. Indeed $\left(G, \tau^{\prime}\right)$ is a $k_{\omega}$-space. We wish to show that $\left(G, \tau^{\prime}\right)$ is a topological group. To do this we must show that the map $f:\left(G, \tau^{\prime}\right) \times\left(G, \tau^{\prime}\right) \rightarrow\left(G, \tau^{\prime}\right)$ given by $f(x, y)=x y^{-1}$ is continuous.

Since $\left(G, \tau^{\prime}\right)$ is a $k_{\omega}$-space, $\left(G, \tau^{\prime}\right) \times\left(G, \tau^{\prime}\right)$ is also a $k_{\omega}$-space. Therefore, to show that $f$ is continuous we only have to show that $f$ is continuous on all compact subsets of $\left(G, \tau^{\prime}\right) \times\left(G, \tau^{\prime}\right)$.

Let $K$ be a compact subset of $\left(G, \tau^{\prime}\right) \times\left(G, \tau^{\prime}\right)$. Then $K \subseteq K_{1} \times K_{1}$, where $K_{1}$ is a compact subset of $\left(G, \tau^{\prime}\right)$. Since $\left(G, \tau^{\prime}\right)$ is a $k_{\omega}$-space with decomposition $G=\bigcup X^{n}$, we see that $K_{1} \subseteq X^{n}$, for some $n$. Thus $f(K) \subseteq$ $f\left(K_{1} \times K_{1}\right) \subseteq f\left(X^{n} \times X^{n}\right) \subseteq X^{2 n}$. Noting that $K$ is compact and $\tau^{\prime} \supseteq \tau$, we see that $K$ has the same induced topology as a subset of $\left(G, \tau^{\prime}\right) \times\left(G, \tau^{\prime}\right)$ as it has as a subset of $(G, \tau) \times(G, \tau)$. Similarly $X^{2 n}$ has the same induced topology as a subset of $\left(G, \tau^{\prime}\right)$ as it has a subset of $(G, \tau)$. Thus, since $(G, \tau)$ is a topological group, $f: K \rightarrow X^{2 n}$ is continuous. So $f$ is continuous on all compact subsets of $\left(G, \tau^{\prime}\right) \times\left(G, \tau^{\prime}\right)$. Hence $\left(G, \tau^{\prime}\right)$ is a topological group.

Now a subset $A$ of $X$ is closed in the topology induced on $X$ from $\left(G, \tau^{\prime}\right)$ if and only if $A=A^{\prime} \cap X$ where $A^{\prime} \cap X^{n}$ is compact for every $n$. Since $X_{n} \subset X^{n}, A^{\prime} \cap X^{n}$ compact implies $A^{\prime} \cap X_{n}$ is compact for every $n$. But $X_{n} \subset X$, so $A^{\prime} \cap X_{n}=A^{\prime} \cap X \cap X_{n}=A \cap X_{n}$ is compact, for each $n$. Now since $X \subset(G, \tau)$ is a $k_{\omega}$-space, $A$ is closed in the topology induced on $X$ from $(G, \tau)$. Noting that $\tau^{\prime} \supseteq \tau$ we then see that $(G, \tau)$ and $\left(G, \tau^{\prime}\right)$ induce the same topology on $X$. However, by hypothesis, $\tau$ is the finest group topology on $G$ inducing the given topology on $X$ and $\tau^{\prime} \supseteq \tau$. Hence $\tau^{\prime}=\tau$.

Thus $G$ is a $k_{\omega}$-space and $A$ is closed in $G$ if and only if $A \cap X^{n}$ is compact, for all $n$.

Now let $A$ be a subset of $G$ such that $A \cap G_{n}(X)$ is closed in $G_{n}(X)$, for all $n$. Noting that $X^{n} \subseteq G_{n}(X)$, we have that $A \cap X^{n}$ is compact, for all $n$. Thus $A$ is closed in $G$. The proof is complete.

Remark. We note that the sets $G_{n}(X)$ (or even $X$ itself) need not be closed subsets of $G$. For example, if $G$ is the additive group of reals and $X=(-1,1)$, then $G_{n}(X)=(-n, n)$ which is not closed even though Theorem 1 clearly applies.

Corollary 1. Let $X$ be a $k_{\omega}$-space and $G$ one of the following: (i) $a$ Markov free topological group on X; (ii) a Markov free abelian topological 
group on $X$; (iii) a Graev free topological group on $X$; (iv) a Graev free abelian topological group on $X$. Then $G$ is a $k_{\omega}$-space and a subset $A$ of $G$ is closed if and only if $A \cap G_{n}(X)$ is closed in $G_{n}(X)$, for all $n$.

Theorem 2. Let $X=\bigcup X_{n}$ be a decomposition of a $k_{\omega}$-space $X$ into compact sets. Let $G$ be a Hausdorff group generated algebraically by $X$ and let $X^{n}=G_{n}\left(X_{n}\right)$. If $G$ has the property that a subset $A$ of $G$ is closed in $G$ if and only if $A \cap X^{n}$ is compact for all $n$, then the topology of $G$ is the finest group topology which induces the given topology on $X$.

Proof. Let $\tau$ be the given topology on $G$ and $\tau^{\prime} \supseteq \tau$ the finest group topology inducing the given topology on $X$. By the proof of Theorem 1, $A \subset G$ is closed in $\left(G, \tau^{\prime}\right)$ if and only if each $A \cap X^{n}$ is compact. But $\tau$ and $\tau^{\prime}$ induce the same topology on $X$, hence on $X_{n}$ and hence also on $X^{n}$. Thus $\tau^{\prime}=\tau$ as desired.

THEOREM 3. Let $X=\bigcup X_{n}$ be a $k_{\omega}$-space. Let $Y \subset F G X$ be a subset such that $Y-\{e\}$ freely generates $G(Y)$, the subgroup of $F G X$ generated by $Y$. Suppose $Y_{1}, Y_{2}, \cdots$ is a sequence of compact subsets of $Y$ such that $Y=$ $\bigcup Y_{n}$ is a $k_{\omega}$-decomposition of $Y$ inducing the same topology on $Y$ that $Y$ inherits as a subset of $F G X$. Put $X^{n}=G_{n}\left(X_{n}\right)$ and $Y^{n}=G_{n}\left(Y_{n}\right)$. If for each natural number $n$ there is an $m$ such that $G(Y) \cap X^{n} \subseteq Y^{m}$, then $G(Y)$ is the Graev free topological group on $Y$ and both $G(Y)$ and $Y$ are closed subsets of FGX.

Proof. It follows from the proof of Theorem 1 that, to prove $G(Y)$ is closed in $F G X$, we only have to show that $G(Y) \cap X^{n}$ is compact for each $n$. Now $G(Y) \cap X^{n}=G(Y) \cap X^{n} \cap Y^{m}=Y^{m} \cap X^{n}$, and hence is compact. Thus $G(Y)$ is closed in $F G X$. Similarly $Y$ is closed in $F G X$.

Using Theorem 2, to prove $G(Y)$ is the Graev free topological group on $Y$, it suffices to show that a subset $A$ of $G(Y)$ is closed if $A \cap Y^{n}$ is compact for all $n$. Consider $A \cap X^{n}$, for any $n$. There exists an $m$ such that $G(Y) \cap$ $X^{n} \subseteq Y^{m}$ and hence $A \cap X^{n}=A \cap X^{n} \cap G(Y)=A \cap X^{n} \cap Y^{m}=\left(A \cap Y^{m}\right) \cap X^{n}$. Since both $A \cap Y^{m}$ and $X^{n}$ are compact, $A \cap X^{n}$ is compact, for all $n$. Thus $A$ is a closed subset of $F G X$ and the proof is complete.

Corollary 2 [2, TheOREM 10]. Let $X$ be a compact Hausdorff space and $Y$ a compact subset of $F G X$ which freely generates $G(Y)$. If for each $n$ there is an $m$ such that $G(Y) \cap G_{n}(X) \subset G_{m}(Y)$, then $G(Y)$ is the Graev free topological group on $Y$ and it is closed in $F G X$.

Proof. Apply Theorem 3 with $X_{n}=X$ and $Y_{n}=Y$ for all $n$. We now generalize Proposition 1.6 of [11]. 
THEOREM 4. Let the Hausdorff group $X$ be a $k_{\omega}$-space. Let $\psi: F G X \rightarrow X$ be the canonical quotient morphism. Then the kernel $K$ of $\psi$ is a Graev free topological group.

Proof. Since $X$ is both a topological group and a $k_{\omega}$-space there exists a sequence $\left\{X_{n}\right\}$ of compact sets such that (i) $X=\bigcup_{n=1}^{\infty} X_{n}$; (ii) $X_{n} \subseteq X_{n+1}$, for all $n$; (iii) if $x \in X_{n}$ and $y \in X_{m}$ then $x y \in X_{n+m}$; (iv) $x \in X_{n}$ implies the inverse (in $X$ ) of $x$ is in $X_{n}$; (v) a subset $A$ of $X$ is closed if and only if $A \cap X_{n}$ is compact, for all $n$.

Define a map $\phi: X \times X \rightarrow F G X$ by $\phi(x, y)=x \cdot y \cdot(x y)^{-1}$ where $x \in X$, $y \in X, x y \in X$ and $(x y)^{-1}$ is the inverse in $F G X$ of $x y$. Define $B=\phi(X \times X)$ and $B_{n}=\phi\left(X_{n} \times X_{n}\right)$. Then each $B_{n}$ is compact and $B=\bigcup_{n=1}^{\infty} B_{n}$. It follows from Hall ([3, pp. 94-98]; see also $[9,1.3-1.6])$ that $K$ is freely generated by $B-\{e\}$. Put $B^{n}=G_{n}\left(B_{n}\right)$ and $X^{n}=G_{n}\left(X_{n}\right)$. We now prove $\left({ }^{*}\right)$.

For each $n$ there is an $m$ such that

$$
K \cap X^{n} \subseteq B^{m}
$$

Let $k \in K$, so $k=x_{1}^{\varepsilon_{1}} \cdots x_{n}^{\varepsilon_{n}}$, where $x_{i} \in X_{n}$ and $\varepsilon_{i}= \pm 1, i=1, \cdots, n$. (Note $x_{i}^{-1}$ denotes the inverse in $F G X$ of $x_{i}$.) It is readily verified that $k=b_{1} \cdots b_{n}$, where

$$
b_{j}=\left(x_{1}^{\varepsilon_{1}} \cdots x_{j-1}^{\varepsilon_{j}-1}\right) \cdot\left(x_{j}\right)^{\varepsilon_{j}} \cdot\left(x_{1}^{\varepsilon_{1}} \cdots x_{j}^{\varepsilon_{j}}\right)^{-1}, \quad j>1
$$

and $b_{1}=x_{1}^{\varepsilon_{1}} \cdot\left(x_{1}^{\varepsilon_{1}}\right)^{-1}$. Here all operations inside parentheses are in $X$ and all operations outside parentheses (inverting the third term and inverting $x_{j}$ in the second term if $\left.\varepsilon_{j}=-1\right)$ are in $F G X$. [Recall that $\left(x_{1}^{\varepsilon_{1}} \cdots x_{n}^{\varepsilon_{n}}\right)$ is the identity element.] We now observe that if $\varepsilon_{j}=+1, b_{j}=\phi\left(x_{1}^{\varepsilon_{1}} \cdots x_{j-1}^{\varepsilon_{j}-1}, x_{j}\right)$, while if $\varepsilon_{j}=-1, b_{j}=\left(\phi\left(x_{1}^{\varepsilon_{1}} \cdots x_{j}^{\varepsilon_{j}}, x_{j}\right)\right)^{-1}$ the inverse being taken in $F G X$. We now note using the properties (iii) and (iv) of the sets $X_{n}$ that, since each $x_{i} \in X_{n}$, each $b_{j}$ is a word of three letters each of which is in (at worst) $X_{n^{2}}$ and thus $b_{j} \in B_{n^{2}}$. But then $k \in G_{n}\left(B_{n^{2}}\right) \subset B^{n^{2}}$, as desired, proving $(*)$.

Our next step is to show that $B$ is closed in $F G X$. Now $B \cap X^{n} \subseteq$ $B \cap\left(K \cap X^{n}\right) \subseteq B \cap B^{m}=B_{m}$. Thus $B \cap X^{n}=B_{m} \cap X^{n}$ which is clearly compact. Hence $B$ is closed in $F G X$. Therefore $B$ is a $k_{\omega}$-space with decomposition $B=\bigcup\left(B \cap X^{n}\right)$. Now for each $n, B \cap X^{n} \subset B_{m}$, for some $m$; conversely each $B_{m}$, being compact, is contained in some $B \cap X^{k}$. Thus the decomposition $B=\bigcup B_{n}$ induces the same topology on $B$ as $B=\bigcup\left(B \cap X^{n}\right)$, which induces the subspace topology on $B \subset F G X$.

Since we have now checked each hypothesis of Theorem $3, K$ is the Graev free topological group on $B$.

COROLlary 3. Let the Hausdorff abelian group $X$ be a $k_{\omega}$-space Let $Z G X$ be the Graev free abelian topological group on $X$ and $\Psi: Z G X \rightarrow X$ the 
canonical quotient morphism. Then the kernel $K$ of $\Psi$ is a Graev free abelian topological group.

Remark. We note that by Remark 2 of [7], Theorem 3 remains true if "Graev" is replaced throughout by "Markov".

COROLlary 4. Let the Hausdorff abelian group $X$ be a $k_{\omega}$-space. Then the projective dimension of $X$ is either zero or one. In particular, the projective dimension of a connected locally compact abelian group or a compact abelian group is one.

Proof. This result follows immediately from Corollary 3 and the fact (Proposition 2.1 of [11]) that no locally compact abelian group is projective unless it is a discrete free abelian group.

\section{REFERENCES}

1. A. V. Arhangel'skiǐ, Mappings related to topological groups, Dokl. Akad. Nauk SSSR 181 (1968), 1303-1306=Soviet Math. Dokl. 9 (1968), 1011-1015. MR 38 \#2237.

2. M. I. Graev, Free topological groups, Izv. Akad. Nauk SSSR Ser. Mat. 12 (1948), 279-324; English transl., Amer. Math. Soc. Transl. (1) 8 (1962), 305-364. MR 10, 11.

3. M. Hall, Jr., The theory of groups, Macmillan, New York, 1959. MR 21 \#1996.

4. A. A. Markov, On free topological groups, Izv. Akad. Nauk SSSR Ser. Mat. 9 (1945), 3-64; English transl., Amer. Math. Soc. Transl. (1) 8 (1962), 195-272. MR 7, 7.

5. E. Michael, Bi-quotient maps and Cartesian products of quotient maps, Ann. Inst. Fourier (Grenoble) 18 (1968), fasc. 2, 287-302; vii (1969). MR 39 \#6277.

6. Sidney A. Morris. Varieties of topological groups. II, Bull. Austral. Math. Soc. 2 (1970), 1-13. MR 41 \#3655b.

7. - Varieties of topological groups and left adjoint functors, J. Austral. Math. Soc. (to appear).

8. - Local compactness and free products of topological groups. II (submitted).

9. - Free products of connected locally compact groups are not sin groups. (submitted).

10. - Free products of Lie groups (submitted).

11. Eric C. Nummela, The projective dimension of a compact abelian group, Proc. Amer. Math. Soc. 38 (1973), 452-456.

12. Edward T. Ordman, Free products of topological groups which are $k_{\omega}$-spaces, Trans. Amer. Math. Soc. (to appear).

13. N. E. Steenrod, A convenient category of topological spaces, Michigan Math. J. 14 (1967), 133-152. MR 35 \#970.

Department of Mathematics, University of Kentucky, Lexington, Kentucky 40506 (Current address of John Mack and Edward T. Ordman)

Department of Mathematics, University of New South Wales, Kensington, N.S.W. 2033, Australia (Current address of Sidney A. Morris) 